\title{
HODOGRAPHIC STUDY OF PLANE MICROPOLAR FLUID FLOWS
}

\author{
INDRASENA ADLURI \\ Department of Mathematics and Computer Science \\ Wheeling Jesuit College \\ Wheeling, West Virginia 23006, U.S.A.
}

(Received January 13, 1993)

\begin{abstract}
Equations of steady flow of a plane micropolar fluid are transformed to the hodograph plane by means of the Legendre transform function of the streamfunction. Results are summarized in the form of a theorem, some flow problems are investigated as applications of this theorem and exact solutions and geometry of the flow are obtained in each case.
\end{abstract}

KEY WORDS AND PHRASES. Micropolar, Legendre, streamfunction. 1991 AMS SUBJECT CLASSIFICATION CODE. 76W05.

\section{INTRODUCTION.}

Flow of a micropolar fluid has been studied by many investigators using the theory and constitutive equations first given and further developed by Eringen ([1], [2]). He presented the theory which is a generalization of the theory of viscous fluids by taking into account the local microrotations and microinertia. The mathematical model underlying micropolar fluid may represent liquid crystals, suspensions, animal blood and the fluids consisting of dumbell molecules. The problem of finding exact solutions of governing equations of micropolar fluid flows presents insurmountable mathematical difficulties due to the fact that these equations are nonlinear. However, exact solutions have been obtained by many researchers in certain particular cases, mostly when the quadratic convective terms vanish in a natural way.

The present study deals with application of hodograph transformation to obtain exact solution of the equations governing the steady plane flow of a micropolar fluid. Chandna and et al ([3]-[9]) have applied hodograph and Legendre transformations to investigate steady plane viscous flows, non-Newtonian flows and constantly inclined, aligned, transverse and orthogonal MHD non-Newtonian flows.

First, the equations of the flow are transformed to the hodograph plane interchanging the role of independent variables $x, y$ and the components of velocity vector field $u, v$, then introducing a Legendre transform function of the streamfunction, all equations in the hodograph plane are expressed in terms of this transform function. These results are put in the form of a theorem and its corollary. Some interesting flow problems of both physical and geometrical importance are studied as applications of the theorem and exact solutions are obtained in each case.

\section{BASIC EQUATIONS.}

The basic equations governing the steady plane flow of micropolar fluid in the absence of 
body forces and body couples are given by

$$
\begin{gathered}
\frac{\partial u}{\partial x}+\frac{\partial v}{\partial y}=0 \\
\rho\left(u \frac{\partial u}{\partial x}+v \frac{\partial u}{\partial y}\right)=-\frac{\partial p}{\partial x}+k \frac{\partial \nu}{\partial y}-(\mu+k) \frac{\partial w}{\partial y} \\
\rho\left(u \frac{\partial v}{\partial x}+v \frac{\partial v}{\partial y}\right)=-\frac{\partial p}{\partial y}-k \frac{\partial \nu}{\partial x}+(\mu+k) \frac{\partial w}{\partial x} \\
\rho j\left(u \frac{\partial \nu}{\partial x}+v \frac{\partial \nu}{\partial y}\right)=-2 k \nu+k w+\gamma\left(\frac{\partial^{2} \nu}{\partial x^{2}}+\frac{\partial^{2} \nu}{\partial y^{2}}\right)
\end{gathered}
$$

where $(u(x, y), v(x, y))$ is the velocity field, $(0,0, \nu(x, y))$, is the microrotation field, $w=v_{x}-u_{y}, p$ is the pressure, $\rho$ is the fluid density, $j$ is the microinertia and $\mu, k, \gamma$ are material constants.

Equations (2.1)-(2.4) is a system of four equations in four unknown functions $u(x, y), v(x, y)$, $\nu(x, y)$ and $p(x, y)$.

Introducing

$$
H(x, y)=\frac{1}{2} \rho\left(u^{2}+v^{2}\right)+p, \nabla^{2}=\frac{\partial^{2}}{\partial x^{2}}+\frac{\partial^{2}}{\partial y^{2}}
$$

equations (2.2)-(2.4) can be rewritten as

$$
\begin{gathered}
\frac{\partial H}{\partial x}=\rho v w+k \frac{\partial \nu}{\partial y}-(\mu+k) \frac{\partial w}{\partial y} \\
\frac{\partial H}{\partial y}=-\rho u w-k \frac{\partial \nu}{\partial x}+(\mu+k) \frac{\partial w}{\partial x} \\
\rho j\left(u \frac{\partial \nu}{\partial x}+v \frac{\partial \nu}{\partial y}\right)=-2 k \nu+k w+\gamma \nabla^{2} \nu .
\end{gathered}
$$

Eliminating $H(x, y)$ from (2.6) and (2.7), we get

$$
\rho\left(u \frac{\partial w}{\partial x}+v \frac{\partial w}{\partial y}\right)=-k \nabla^{2} \nu+(\mu+k) \nabla^{2} w
$$

\section{EQUATIONS IN THE HODOGRAPH PLANE.}

Let the flow variables $u(x, y), v(x, y)$ be such that, in the region of flow, the Jacobian

$$
J(x, y)=\frac{\partial(u, v)}{\partial(x, y)} \neq 0, \quad 0<|J|<\infty .
$$

Considering $x, y$ as functions of $u, v$, we can derive the following relations:

$$
\begin{gathered}
\frac{\partial u}{\partial x}=J \frac{\partial y}{\partial v}, \quad \frac{\partial u}{\partial y}=-J \frac{\partial x}{\partial v}, \quad \frac{\partial v}{\partial x}=-J \frac{\partial y}{\partial u}, \quad \frac{\partial v}{\partial y}=J \frac{\partial x}{\partial u} \\
J(x, y)=\frac{\partial(u, v)}{\partial(x, y)}=\left[\frac{\partial(x, y)}{\partial(u, v)}\right]^{-1}=\bar{J}(u, v) \\
\frac{\partial f}{\partial x}=\frac{\partial(f, y)}{\partial(x, y)}=J \frac{\partial(\bar{f}, y)}{\partial(u, v)}=\bar{J} \frac{\partial(\bar{f}, y)}{\partial(u, v)} \\
\frac{\partial f}{\partial y}=-\frac{\partial(f, x)}{\partial(x, y)}=J \frac{\partial(\bar{x}, f)}{\partial(u, v)}=\bar{J} \frac{\partial(x, \bar{f})}{\partial(u, v)}
\end{gathered}
$$


where $f(x, y)=f(x(u, v), y(u, v))=\bar{f}(u, v)$ is any continuously differentiable function.

Using the above relations, we can transform (2.1), (2.9) and (2.8), respectively, into the following system of equations in the $(u, v)$-plane

$$
\begin{gathered}
\frac{\partial x}{\partial u}+\frac{\partial y}{\partial v}=0 \\
\rho\left(v P_{1}+u P_{2}\right)=-k\left\{\frac{\partial\left(x, \bar{J} Q_{1}\right)}{\partial(u, v)}+\frac{\partial\left(\bar{J} Q_{2}, y\right.}{\partial(u, v)}\right\} \\
+(\mu+k)\left\{\frac{\partial\left(x, \bar{J} P_{1}\right)}{\partial(u, v)}+\frac{\partial\left(\bar{J} P_{2}, y\right)}{\partial(u, v)}\right\} \\
\rho j \bar{J}\left(v Q_{1}+u Q_{2}\right)=-2 k \bar{\nu}+k \bar{w}+\gamma \bar{J}\left\{\frac{\partial\left(x, \bar{J} Q_{1}\right)}{\partial(u, v)}+\frac{\partial\left(\bar{J} Q_{2}, y\right)}{\partial(u, v)}\right\}
\end{gathered}
$$

where

$$
\begin{array}{cc}
w(x, y)=w(x(u, v), & y(u, v))=\bar{w}(u, v) \\
\nu(x, y)=\nu(x(u, v), & y(u, v))=\bar{\nu}(u, v) \\
P_{1}=P_{1}(u, v)=\frac{\partial(x, \bar{w})}{\partial(u, v)}, & P_{2}=P_{2}(u, v)=\frac{\partial(\bar{w}, y)}{\partial(u, v)} \\
Q_{1}=Q_{1}(u, v)=\frac{\partial(x, \bar{\nu})}{\partial(u, v)}, & Q_{2}=Q_{2}(u, v)=\frac{\partial(\bar{\nu}, y)}{\partial(u, v)} .
\end{array}
$$

Equations (3.4)-(3.6) is a system of three equations in three unknown functions $x(u, v), y(u, v)$ and $\bar{\nu}(u, v)$. Once this system is solved for $x(u, v), y(u, v)$ and $\bar{\nu}(u, v)$, we can determine $u(x, y), v(x, y), w(x, y), \nu(x, y)$ and $p(x, y)$ for the system of equations (2.1)-(2.4) governing the steady plane flow of a micropolar fluid.

\section{EQUATIONS IN LEGENDRE TRANSFORM FUNCTION AND $\bar{\nu}(u, v)$.}

The equation of continuity (2.1) implies the existence of a streamfunction $\psi(x, y)$ such that

$$
d \psi=-v d x+u d y \text { or } \frac{\partial \psi}{\partial x}=-v, \frac{\partial \psi}{\partial y}=u
$$

and equation (3.4) implies the existence of a function $L(u, v)$, called a Legendre transform function of the streamfunction $\psi(x, y)$, so that

$$
d L=-y d u+x d v \text { or } \frac{\partial L}{\partial u}=-y, \frac{\partial L}{\partial v}=x
$$

Functions $\psi(x, y)$ and $L(u, v)$ are related by

$$
L(u, v)=v x-u y+\psi(x, y)
$$

Using (3.2), (3.3) and (4.2), equations (3.5) and (3.6) can be transformed, respectively, into the following equations

$$
\begin{gathered}
\rho\left(v P_{1}+u P_{2}\right)=-k\left\{\frac{\partial\left(\partial L / o v, \bar{J} Q_{1}\right)}{\partial(u, v)}+\frac{\partial\left(\partial L / \partial u, \bar{J} Q_{2}\right)}{\partial(u, v)}\right\} \\
+(\mu+k)\left\{\frac{\partial\left(\partial L / \partial v, \bar{J} P_{1}\right)}{\partial(u, v)}+\frac{\partial\left(\partial L / \partial u, \bar{J} P_{2}\right)}{\partial(u, v)}\right\}
\end{gathered}
$$


where

$$
\rho J \bar{J}\left(v Q_{1}+u Q_{2}\right)=-2 k \bar{\nu}+k \bar{w}+\gamma \bar{J}\left\{\frac{\partial\left(\partial L / \partial v, \bar{J} Q_{1}\right)}{\partial(u, v)}+\frac{\partial\left(\partial L / \partial u, \bar{J} Q_{2}\right)}{\partial(u, v)}\right\}
$$

$$
\begin{array}{cc}
\bar{J}=\left[\frac{\partial^{2} L}{\partial v^{2}} \cdot \frac{\partial^{2} L}{\partial u^{2}}-\left(\frac{\partial^{2} L}{\partial u \partial v}\right)^{2}\right]^{-1} & \\
\bar{w}=\bar{J}\left(\frac{\partial^{2} L}{\partial u^{2}}+\frac{\partial^{2} L}{\partial v^{2}}\right) & P_{2}(u, v)=\frac{\partial(\partial L / \partial u, \bar{w})}{\partial(u, v)} \\
P_{1}(u, v)=\frac{\partial(\partial L / \partial v, \bar{w})}{\partial(u, v)}, & Q_{2}(u, v)=\frac{\partial(\partial L / \partial u, \bar{\nu})}{\partial(u, v)} .
\end{array}
$$

We can summarize the above results in the form of the following theorem.

THEOREM. If $L(u, v)$ is the Legendre transform of a streamfunction of a steady plane flow of a micropolar fluid and $\bar{\nu}(u, v)$ is the transformed microrotation function, then $L(u, v)$ and $\bar{\nu}(u, v)$ must satisfy (4.4) and (4.5) where $\bar{J}(u, v), \bar{w}(u, v), P_{1}(u, v), P_{2}(u, v), Q_{1}(u, v)$ and $Q_{2}(u, v)$ are given by (4.6)-(4.9).

Once $L(u, v)$ and $\bar{\nu}(u, v)$ are obtained, the velocity components are found from (4.2). Using the velocity components $u(x, y), v(x, y)$, we can determine $p(x, y)$ and $\nu(x, y)$ in the physical plane.

Now, we express (4.4)-(4.9) in terms of polar coordinates $(q, \theta)$ in the hodograph plane by defining

$$
q^{2}=u^{2}+v^{2}, \quad \theta=\tan ^{-1}(v / u)
$$

By partial differentiation, we can get

$$
\begin{gathered}
\frac{\partial f}{\partial u}=\cos \theta \frac{\partial f^{*}}{\partial q}-\frac{\sin \theta}{q} \frac{\partial f^{*}}{\partial \theta}, \quad \frac{\partial f}{\partial v}=\sin \theta \frac{\partial f^{*}}{\partial q}+\frac{\cos \theta}{q} \frac{\partial f^{*}}{\partial \theta} \\
\frac{\partial(f, g)}{\partial(u, v)}=\frac{\partial\left(f^{*}, g^{*}\right)}{\partial(q, \theta)} \cdot \frac{\partial(q, \theta)}{\partial(u, v)}=\frac{1}{q} \frac{\partial\left(f^{*}, g^{*}\right)}{\partial(q, \theta)}
\end{gathered}
$$

where $f(u, v)=f^{*}(q, \theta), g(u, v)=g^{*}(q, \theta)$ are any continuously differentiable functions.

Denoting $L^{*}(q, \theta), \nu^{*}(q, \theta), J^{*}(q, \theta), w^{*}(q, \theta)$ to be the respectively the transformed functions of $f(u, v), \bar{\nu}(u, v), \bar{J}(u, v), \bar{w}(u, v)$ in $(q, \theta)$ coordinates, we can write (4.4)-(4.9) in polar coordinates as follows:

$$
\begin{gathered}
\rho\left(v P_{1}^{*}+u P_{2}^{*}\right)=-\frac{k}{q}\left[\frac{\partial\left(\sin \theta \frac{\partial L^{*}}{\partial q}+\frac{\cos \theta}{q} \frac{\partial L^{*}}{\partial \theta}, J^{*} Q_{1}^{*}\right)}{\partial(q, \theta)}\right. \\
\left.+\frac{\partial\left(\cos \theta \frac{\partial L^{*}}{\partial q}-\frac{\sin \theta}{q} \frac{\partial L^{*}}{\partial \theta}, J^{*} Q_{2}^{*}\right)}{\partial(q, \theta)}\right]+\frac{(\mu+k)}{q}\left[\frac{\partial\left(\sin \theta \frac{\partial L^{*}}{\partial q}+\frac{\cos \theta}{q} \frac{\partial L^{*}}{\partial \theta}, P_{1}^{*}\right)}{\partial(q, \theta)}\right. \\
\left.+\frac{\partial\left(\cos \theta \frac{\partial L^{*}}{\partial q}-\frac{\sin \theta}{q} \frac{\partial L^{*}}{\partial \theta}, P_{2}^{*}\right)}{\partial(q, \theta)}\right] \\
\rho j J^{*}\left(v Q_{1}^{*}+u Q_{2}^{*}\right)=-2 k \nu^{*}+k w^{*}+\frac{\gamma J^{*}}{q}\left[\frac{\partial\left(\sin \theta \frac{\partial L^{*}}{\partial q}+\frac{\cos \theta}{q} \frac{\partial L^{*}}{\partial \theta}, J^{*} Q_{1}^{*}\right)}{\partial(q, \theta)}\right. \\
\left.+\frac{\left.\partial\left(\cos \theta \frac{\partial L^{*}}{\partial q}-\frac{\sin \theta}{q} \frac{\partial L^{*}}{\partial \theta}, J_{2}^{*} Q_{2}^{*}\right)\right]}{\partial(q, \theta)}\right]
\end{gathered}
$$




$$
\begin{gathered}
J^{*}=q^{4}\left[q^{2} \frac{\partial^{2} L^{*}}{\partial q^{2}}\left(q \frac{\partial L^{*}}{\partial q}+\frac{\partial^{2} L^{*}}{\partial \theta^{2}}\right)-\left(\frac{\partial L^{*}}{\partial \theta}-q \frac{\partial^{2} L^{*}}{\partial q \partial \theta}\right)^{2}\right]^{-1} \\
w^{*}(q, \theta)=J^{*}\left(\frac{\partial^{2} L^{*}}{\partial q^{2}}+\frac{1}{q^{2}} \frac{\partial^{2} L^{*}}{\partial \theta^{2}}+\frac{1}{q} \frac{\partial L^{*}}{\partial q}\right) \\
P_{1}^{*}(q, \theta)=\frac{1}{q} \frac{\partial\left(\sin \theta \frac{\partial L^{*}}{\partial q}+\frac{\cos \theta}{q} \frac{\partial L^{*}}{\partial \theta}, w^{*}\right)}{\partial(q, \theta)} \\
P_{2}^{*}(q, \theta)=\frac{1}{q} \frac{\partial\left(\cos \theta \frac{\partial L^{*}}{\partial q}-\frac{\sin \theta}{q} \frac{\partial L^{*}}{\partial \theta}, w^{*}\right)}{\partial(q, \theta)} \\
Q_{1}^{*}(q, \theta)=\frac{1}{q} \frac{\partial\left(\sin \theta \frac{\partial L^{*}}{\partial q}+\frac{\cos \theta}{q} \frac{\partial L^{*}}{\partial \theta}, \nu^{*}\right)}{\partial(q, \theta)} \\
Q_{2}^{*}(q, \theta)=\frac{1}{q} \frac{\partial\left(\cos \theta \frac{\partial L^{*}}{\partial q}-\frac{\sin \theta}{q} \frac{\partial L^{*}}{\partial \theta}, \nu^{*}\right)}{\partial(q, \theta)}
\end{gathered}
$$

Once $L^{*}(q, \theta)$ and $\nu^{*}(q, \theta)$ are known, we can determine $u(x, y), v(x, y)$, and other flow variables in the physical plane.

From (4.10)-(4.17), we can have the following corollary.

COROLLARY. If $L^{*}(q, \theta)$ is the Legendre transform function of a steady plane flow of a micropolar fluid and $\nu^{*}(q, \theta)$ is the transformed microrotation function, then $L^{*}(q, \theta)$ and $\nu^{*}(q, \theta)$ must satisfy (4.10)-(4.17).

\section{APPLICATIONS.}

In this section, we study various flow problems as applications of the theorem and corollary.

Application 1.

Let

$$
L(u, v)=A u^{2}+B v^{2}+C u+D v+E
$$

be the Legendre transform function, $A, B, C, D, E$ are arbitrary constants, and $A, B$ are nonzero.

Using (5.1) in (4.6)-(4.9), we obtain

$$
\begin{gathered}
\bar{J}=\frac{1}{4 A B}, \quad \bar{w}=\frac{A+B}{2 A \bar{B}}, \quad P_{1}=0, \quad P_{2}=0 \\
Q_{1}=-2 B \frac{\partial \bar{\nu}}{\partial u}, \quad Q_{2}=2 A \frac{\partial \bar{\nu}}{\partial v}
\end{gathered}
$$

Employing (5.1) and (5.2), equations (4.4) and (4.5) yield the following system of equations for $\bar{\nu}(u, v)$

$$
\begin{gathered}
B^{2} \frac{\partial^{2} \bar{\nu}}{\partial u^{2}}+A^{2} \frac{\partial^{2} \bar{\nu}}{\partial v^{2}}=0 \\
\frac{\rho j}{2 A B}\left(A u \frac{\partial \bar{\nu}}{\partial v}-B v \frac{\partial \bar{\nu}}{\partial u}\right)=-2 k \bar{\nu}+\frac{k(A+B)}{2 A B}
\end{gathered}
$$

Solving (5.3), we get

$$
\bar{\nu}(u, v)=c_{1}\left(u-\frac{4 B k}{\rho j} v\right)+\frac{(A+B)}{4 A B}
$$




$$
A B=-\frac{\rho^{2} \jmath^{2}}{16 k^{2}}
$$

where $c_{1}$ is a nonzero arbitrary constant.

Condition (5.5) implies that both $A$ and $B$ are nonzero, $A \neq B$, and have opposite signs.

Substituting (5.1) in (4.2) and solving the resulting equations, we get

$$
u(x, y)=-\frac{(y+C)}{2 A}, v(x, y)=\frac{(x-D)}{2 B}
$$

Using (5.6) in (5.4), (2.6), (2.7) and (4.3), we obtain $\nu(x, y), p(x, y)$ and $\psi(x, y)$ as follows

$$
\begin{gathered}
\nu(x, y)=\frac{c_{1}}{2 A}\left\{\frac{\rho j}{4 k B}(x-D)-(y+C)\right\}+\frac{k(A+B)}{4 A B} \\
p(x, y)=\frac{\rho}{8 A B}\left\{(x-D)^{2}+(y+C)^{2}\right\}-\frac{k c_{1} x}{2 A}-\frac{c_{1} \rho j y}{8 A B}+P_{o} \\
\psi(x, y)=-\frac{1}{4}\left\{\frac{(x-D)^{2}}{B}+\frac{(y+C)^{2}}{A}\right\}+E
\end{gathered}
$$

where $P_{o}$ is an arbitrary constant.

If $L(u, v)=A u^{2}+B v^{2}+C u+D v+E$ is the Legendre transform function of a steady plane flow of a micropolar fluid, then the flow in the physical plane is a flow with hyperbolic stream lines, and the flow variables are given by (5.6)-(5.8) where $A$ and $B$ are nonzero, $A \neq B$ and have opposite signs. From the condition $A \neq B$, and (5.6) it follows that a steady plane flow of a micropolar fluid cannot be a vortex flow.

\section{Application II.}

Let

$$
L(u, v)=(A u+B) v+C u^{2}+D u+E
$$

be the Legendre transform function, where $A(\neq 0), B, C, D, E$ are arbitrary constants.

Employing (5.10) in (4.6)-(4.9), we get

$$
\begin{gathered}
\bar{J}=-\frac{1}{A^{2}}, \bar{w}=-\frac{2 C}{A^{2}}, P_{1}=0, P_{2}=0 \\
Q_{1}=A \frac{\partial \bar{\nu}}{\partial v}, \quad Q_{2}=2 C \frac{\partial \bar{\nu}}{\partial v}-A \frac{\partial \bar{\nu}}{\partial u}
\end{gathered}
$$

Using (5.10) and (5.11) in (4.4) and (4.6), we obtain the following system of equations

$$
\begin{gathered}
\frac{\partial^{2} \bar{\nu}}{\partial u^{2}}-\frac{4 C}{A} \frac{\partial^{2} \bar{\nu}}{\partial u \partial v}+\left(1+\frac{4 C^{2}}{A^{2}}\right) \frac{\partial^{2} \bar{\nu}}{\partial v^{2}}=0 \\
\frac{\rho j}{A^{2}}\left\{(A v+2 C u) \frac{\partial \bar{\nu}}{\partial v}-A u \frac{\partial \bar{\nu}}{\partial u}\right\}=2 k \bar{\nu}+\frac{2 k C}{A^{2}}
\end{gathered}
$$

Solving these equations, we get

$$
\begin{gathered}
\bar{\nu}(u, v)=c_{2}\left(u+\frac{A}{C} v\right)-\frac{C}{A^{2}} \\
A=\frac{\rho j}{2 k}
\end{gathered}
$$

where $c_{2}$ is an arbitrary constant.

Proceeding as in the previous section, we can obtain the following solutions

$$
\begin{gathered}
u(x, y)=\frac{(x-B)}{A}, v(x, y)=-\frac{2 C x+A y+A D-2 B C}{A^{2}} \\
\nu(x, y)=-c_{2}\left\{\frac{(x-B)}{A}+\frac{(y+D)}{C}\right\}-\frac{C}{A^{2}}
\end{gathered}
$$




$$
\begin{gathered}
p(x, y)=\frac{2 C \rho}{A^{4}}\left\{C x^{2}+(A D-2 B C) x+A(x-B) y\right\}-\frac{1}{2} \rho\left\{\frac{(x-B)^{2}}{A^{2}}\right. \\
\left.+\frac{(2 C x+A y+A D-2 B C)^{2}}{A^{4}}\right\}-k c_{2}\left(\frac{x}{C}-\frac{y}{A}\right)+\pi_{o} \\
\psi(x, y)=\frac{C}{A}(x-B)\left\{\frac{(x-B)}{A}+\frac{(y+D)}{C}\right\}+E
\end{gathered}
$$

where $\pi_{o}$ is an arbitrary constant.

If $L(u, v)=(A u+B) v+C u^{2}+D u+E$ is the Legendre transform of a streamfunction of a steady plane flow of a micropolar fluid, then the flow variables are given by (5.15)-(5.18) when $A=\rho j / 2 k$, and the flow in the physical plane

(a) a flow with $C x^{2}+A x y+(A D-2 B C) x=$ constant as streamlines when $C \neq 0$ in $L(u, v)$,

(b) a flow with rectangular hyperbolas, $(x-B)(y+D)=$ constant as streamlines when $C=0$ in $L(u, v)$.

\section{Application III (Spiral Flow).}

Let

$$
L^{*}(q, \theta)=C_{1} \ln q+C_{2} \theta, \quad C_{1} \neq 0, C_{2} \neq 0
$$

be the Legendre transform function of a streamfunction, where $C_{1}$ and $C_{2}$ are arbitrary constants.

Using (5.19), we can evaluate $J^{*}, w^{*}, P_{1}^{*}, P_{2}^{*}, Q_{1}^{*}$ and $Q_{2}^{*}$ in the following form

$$
\begin{gathered}
J^{*}=-\frac{q^{4}}{\left(C_{1}^{2}+C_{2}^{2}\right)}, \quad w^{*}=0, \quad P_{1}^{*}=0, \quad P_{2}^{*}=0 \\
Q_{1}^{*}=-\frac{1}{q^{3}}\left(C_{1} \sin \theta+C_{2} \cos \theta\right) \frac{\partial \nu^{*}}{\partial \theta}-\frac{1}{q^{2}}\left(C_{1} \cos \theta-C_{2} \sin \theta\right) \frac{\partial \nu^{*}}{\partial q} \\
Q_{2}^{*}=-\frac{1}{q^{3}}\left(C_{1} \cos \theta-C_{2} \sin \theta\right) \frac{\partial \nu^{*}}{\partial \theta}+\frac{1}{q^{2}}\left(C_{1} \sin \theta+C_{2} \cos \theta\right) \frac{\partial \nu^{*}}{\partial q} .
\end{gathered}
$$

Employing (5.19) and (5.20) in (4.10) and (4.11), we obtain, respectively,

$$
\begin{gathered}
\frac{\partial^{2} \nu^{*}}{\partial q^{2}}+\frac{1}{q} \frac{\partial \nu^{*}}{\partial q}+\frac{1}{q^{2}} \frac{\partial^{2} \nu^{*}}{\partial \theta^{2}}=0 \\
\frac{\rho j q^{2}}{\left(C_{1}^{2}+C_{2}^{2}\right)}\left\{C_{1} \frac{\partial \nu^{*}}{\partial \theta}-C_{2} q \frac{\partial \nu^{*}}{\partial q}\right\}=-2 k \nu^{*}
\end{gathered}
$$

Solving (5.21) and (5.22), we get

$$
\nu^{*}=0
$$

which is a trivial solution. Therefore, a steady plane flow of a micropolar fluid cannot be a spiral flow.

Application IV (Radial Flow).

Letting $L^{*}(q, \theta)=A_{1} \theta+A_{2}, \quad A_{1} \neq 0$

and proceeding as in the previous section, we obtain

$$
\begin{gathered}
J^{*}=-\frac{q^{4}}{A_{1}^{2}}, \quad w^{*}=0, \quad P_{1}^{*}=0, \quad P_{2}^{*}=0 \\
Q_{1}^{*}=\frac{A_{1}}{q^{2}}\left(\sin \theta \frac{\partial \nu^{*}}{\partial q}-\frac{\cos \theta}{q} \frac{\partial \nu^{*}}{\partial \theta}\right) \\
Q_{2}^{*}=\frac{A_{1}}{q^{2}}\left(\cos \theta \frac{\partial \nu^{*}}{\partial q}+\frac{\sin \theta}{q} \frac{\partial \nu^{*}}{\partial \theta}\right)
\end{gathered}
$$




$$
\begin{gathered}
\frac{\partial^{2} \nu^{*}}{\partial q^{2}}+\frac{1}{q} \frac{\partial \nu^{*}}{\partial q}+\frac{1}{q^{2}} \frac{\partial^{2} \nu^{*}}{\partial \theta^{2}}=0 \\
\frac{\rho j q^{3}}{A_{1}} \frac{\partial \nu^{*}}{\partial q}=2 k \nu^{*}
\end{gathered}
$$

where $A_{1}$ and $A_{2}$ are arbitrary constants.

From (5.26) and (5.27), we get

$$
\nu^{*}=0
$$

which is again a trivial solution. Therefore, a radial flow is not possible in a plane micropolar fluid.

\section{REFERENCES}

1. ERINGEN, A.C., Simple micro-fluids, Int. Engng Sci. 2 (1964), 205-217.

2. ERINGEN, A.C., Theory of micropolar fluids, J. Math. Mech. 16 (1966), 1-18.

3. CHANDNA, O.P.; BARRON, R.M. \& SMITH, A.C., Rotational plane steady flow of viscous fluid, SIAM J. Appl. Math 42 (1982), 1323-1336.

4. SIDDIQUI, A.M.; KALONI, P.N. \& CHANDNA, O.P., Hodograph transformation methods in non-Newtonian fluid flows, J. Engng Math. (1985), 203-216.

5. CHANDNA, O.P. \& NGUYEN, P.V., Hodograph method in non-Newtonian MHD transverse fluid flows, J. Engng Math. 23 (1989), 119-139.

6. NGUYEN, P.V. \& CHANDNA, O.P., Hodographic study of non-Newtonian MHD aligned steady plane flows, Internat. J. Math. and Math. Sci. 13 (1990), 93-114.

7. CHANDNA, O.P. \& NGUYEN, P.V., Hodograph transformation method and solution in aligned MHD plane flows, Int. J. Engng Sci. 28 (1990), 973-987.

8. CHANDNA, O.P. \& NGUYEN, P.V., Hodograph study of MHD constantly inclined fluid flows, Int. J. Engng Sci. 30 (1992), 69-82.

9. NGUYEN, P.V. \& CHANDNA, O.P., Hodograph method in MHD orthogonal fluid flows, Internat. J. Math. and Math. Sci. 15 (1992), 149-160. 


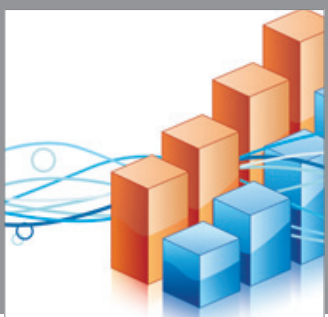

Advances in

Operations Research

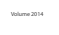

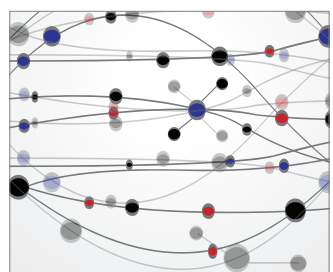

\section{The Scientific} World Journal
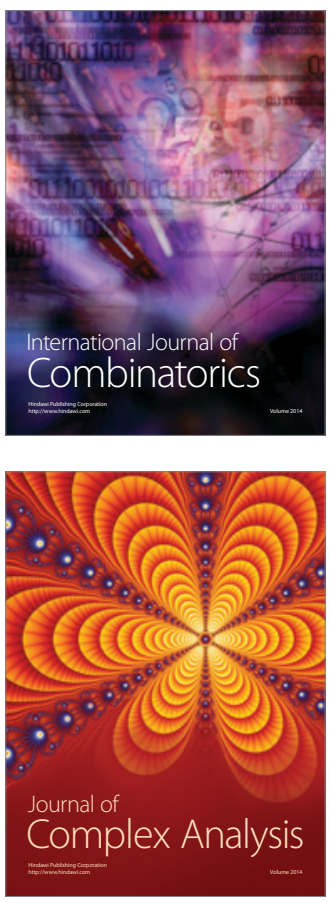

International Journal of

Mathematics and

Mathematical

Sciences
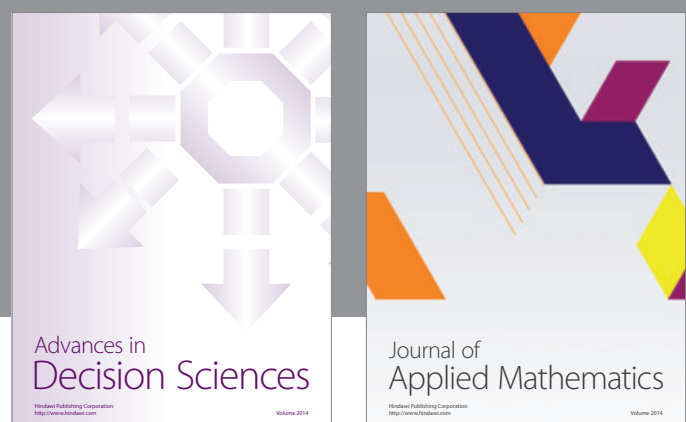

Journal of

Applied Mathematics
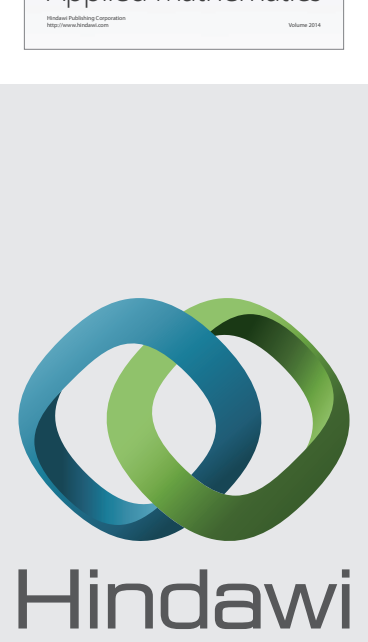

Submit your manuscripts at http://www.hindawi.com
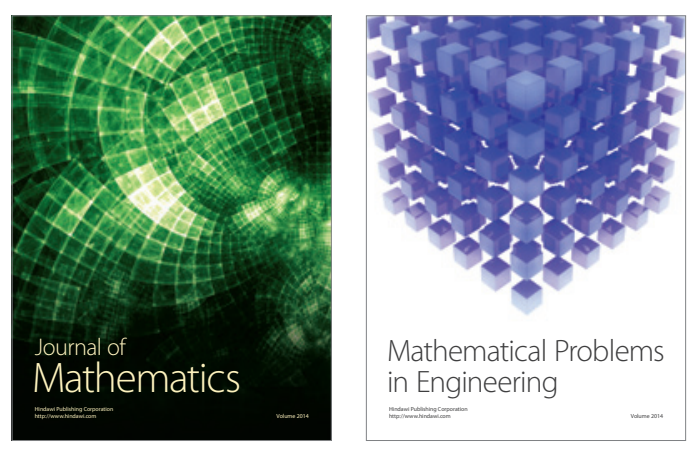

Mathematical Problems in Engineering
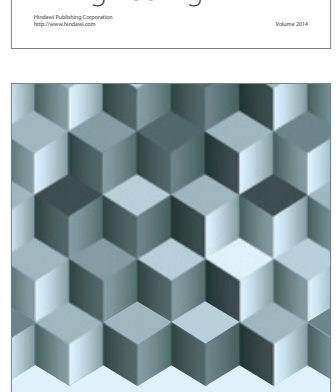

Journal of

Function Spaces
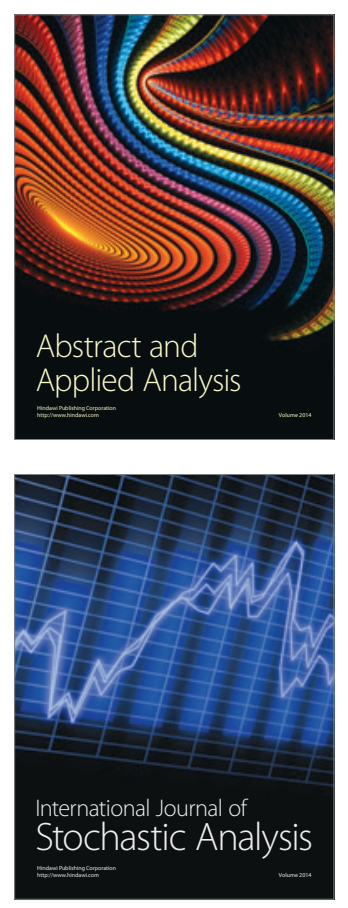

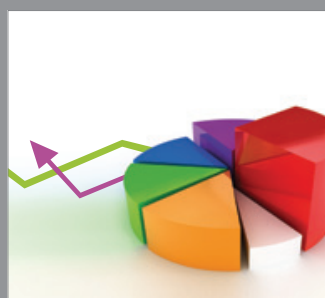

ournal of

Probability and Statistics

Promensencen
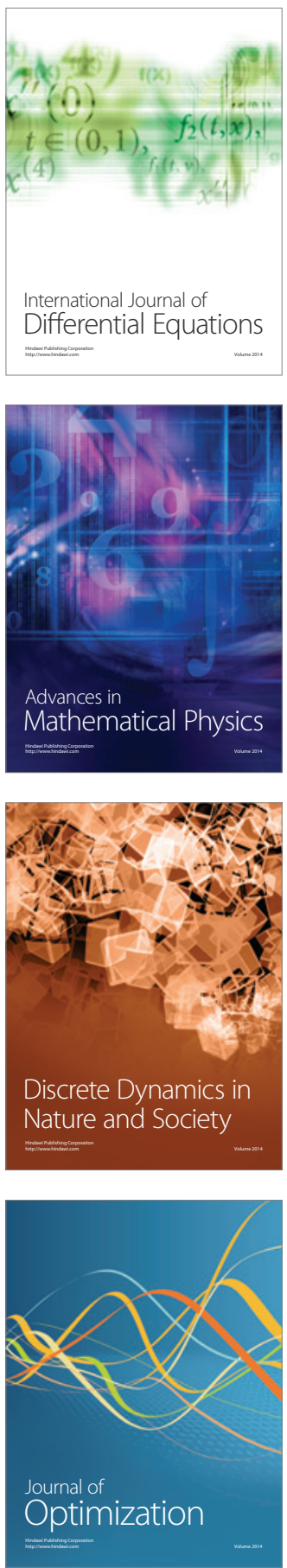